\title{
Modeling periodic adiabatic shear band evolution during high speed machining Ti-6Al-4V alloy
}

\author{
G.G. Ye ${ }^{\text {a,b }}$, S.F. Xue ${ }^{\mathrm{b}}$, M.Q. Jiang ${ }^{\mathrm{a}}$, X.H. Tong ${ }^{\mathrm{b}}$, L.H. Dai ${ }^{\mathrm{a}, *}$ \\ ${ }^{a}$ State Key Laboratory of Nonlinear Mechanics, Institute of Mechanics, Chinese Academy of Sciences, Beijing 100190, China \\ ${ }^{\mathrm{b}}$ Department of Engineering Mechanics, China University of Petroleum, Shandong 266580, China
}

\section{A R T I C L E I N F O}

\section{Article history:}

Received 27 April 2012

Received in final revised form 7 July 2012

Available online 17 July 2012

\section{Keywords:}

High speed machining

Thermo-plastic instability

Segment spacing

Momentum diffusion

Shear band

\begin{abstract}
A B S T R A C T
Cutting experiments were performed on Ti-6Al-4V alloy over a wide range of cutting speeds. The transition of chip morphology from continuous to serrated is observed with increasing the cutting speeds, which is found to be ascribed to a periodic shear band formation that caused by thermo-plastic instability occurred within the primary shear zone (PSZ). Further microscopic observations reveal that the spacing of these periodic shear bands, i.e., the segment spacing, is significantly related to the evolution degree of shear band which increases with increasing the cutting speed. Since the segment spacing is the most important parameter to characterize the chip serration, to predict the segment spacing is fundamentally useful for the understating of serrated chip formation mechanism. However, the complicated conditions of high speed machining (HSM) give rise to greater difficulties for the prediction of segment spacing, and there is still no theoretical prediction has yet considered the effect of shear band evolution. In this work, by analyzing the plastic deformation within the PSZ, and taking into account the evolution of shear band as well as the material convection caused by chip flow, a new theoretical model is developed to predict the segment spacing, in which the momentum diffusion due to unloading within the shear band had been considered. The predictions of this model were compared with the experimental and simulated results, which clearly reveal that the proposed model can satisfactorily capture the process of chip segmentation over a wide range of cutting speeds.
\end{abstract}

Crown Copyright @ 2012 Published by Elsevier Ltd. All rights reserved.

\section{Introduction}

Machining operations are widely employed in industry to remove unwanted materials from a workpiece and obtain designed geometrical dimensions and surface finish. One of the principal by-products of machining operations is chip, and thus the operations are often referred to as chip formation processes, during which the workpiece material undergoes large plastic deformation (Oxley, 1989; Shaw, 2005). The character of chip is of central importance since the chips are witnesses of physical and thermal phenomena happening during such operations (Shaw, 2005). The two basic types of chips are continuous and discontinuous or serrated. Usually, higher cutting speed renders the formation of serrated chip (Barry and Byrne, 2002; Ye et al., 2012). The production of serrated chip ties up with decreased tool life, degradation of the workpiece surface finish and less accuracy in the machined part (Davies et al., 1996). Therefore, to understand the cause of serrated chip formation and further predict its characteristic size, such as the segment spacing, is of practical importance.

\footnotetext{
* Corresponding author.

E-mail address: lhdai@Inm.imech.ac.cn (L.H. Dai).
} 
There are two controversial theories for the serrated chip formation. The first attributes the serrated chip formation to a repeated thermo-plastic instability occurred within the primary shear zone (PSZ) (Recht, 1964; Komanduri, 1982, 1984; Davies et al., 1997; Molinari et al., 2002; Ma et al., 2012). The second ascribes it to a periodic crack initiated in the free surface of workpiece ahead of the tool (Nakayama et al., 1988; Shaw and Vyas, 1993; Elbestawi et al., 1996; Vyas and Shaw, 1999; Astakhov, 2010). For ductile materials, the serrated chip formation is usually characterized by periodic adiabatic shear bands (ASBs) formed within the PSZ (Barry and Byrne, 2002; Astakhov, 1998, 2010; Sheikh-Ahmad et al., 2004). The ASB is an especial plastic deformation phenomenon, which is one of the most important deformation and failure mechanisms in materials subjected to high-strain-rate loading. Because of its technological importance, the ASB poses an interesting topic to researchers, and a large amount of literatures on ASB by Bai and Dodd (1992), Grady (1992), Aifantis (1987), Zbib and Aifantis (1988, 1992, 2003), Zbib and Jubran (1992), Meyers (1994), Wright and Ockendon (1996), Molinari (1997), Batra and Wei (2006), Kuroda (1996), Kuroda and Tvergaard (2007), Rittel et al. (2006, 2008) are available. In recent years, the formation and the mechanism of ASB have still received considerable attention. Kobayashi (2010) analyzed the shear banding behaviors based on the theory of ultrasonic wave propagating in plastic materials, and obtained the diagrams of diffuse necking, localized necking and the formation of limit conditions. Meredith and Khan (2012) investigated the macroscopic shear band failure in titanium subjected to dynamic loading after equal channel angular pressing (ECAP, which is somewhat same as the continuous chip formation process), and fund that the onset was dependent on the loading direction and testing temperature. The study by Luo et al. (2012) on the onset of shear banding and fracture of anisotropic aluminum under multi-axial loading also showed a strong dependency of strain to localization on the material orientation with respect to the loading direction. They further proposed an uncoupled non-associated anisotropic fracture model to predict the onset of shear banding. Focusing on the rate-independent isotropic materials, Anand et al. (2012) presented a large deformation gradient theory to model the large-deformation strain-softening response accompanied by intense localized shear bands. Also, Kumar and Mahesh (2012) developed a rigid-plastic rate-independent crystal plasticity model to predict the macroscopic banding response, lattice orientation evolution, slip distribution and band boundary evolution in single crystals subjected to homogeneous macroscopic deformation. In the study of localized failure mode, based on the magnitude of stress vector, Khan and Liu (2012a,b) established a universal, accurate and efficient fracture criterion for ductile metals, which was further developed to include strain rate and temperature dependences. Moreover, the investigations on shear banding behaviors showed a tendency toward extended material models recently. For example, some studies focused on the formation of shear bands in bulk metallic glasses (Jiang and Dai, 2009a, 2009b, 2011; Henann and Anand, 2009; Chen and Lin, 2010; Wu et al., 2011), and others dealt with the localized failure modes of porous media (Mroginski et al., 2011).

As for metal/alloy cutting, considerable efforts have also been carried out to focus on the adiabatic shear localization in the serrated chip formation process during past decades, and several classical mechanical models have been developed to derive conditions under which continuous chip first becomes unstable. A pioneering study was carried out by Recht (1964) to provide the first explanation for the transition from continuous to serrated chip formation in machining. He pointed out that, when the tendency of a material to harden with plastic deformation is overtaken by thermal softening effects, periodic catastrophic thermoplastic shear bands can form in the workpiece material. von-Turkovich and Durham (1982) used a similar approach to explain the transition in the chip formation from continuous to serrated. It was assumed that such a transition would occur only for materials which exhibit a flow stress maximum in the stress-strain curve at shear strains, strain rates and temperatures comparable to those occurring in the deformed chip. The analytical model presented by Semiatin and Rao (1983) is perhaps the first to provide a quantitative prediction of the critical speed at which the serrated chips are produced. This model incorporates a simple heat-transfer analysis and materials properties such as the strainhardening, temperature dependence, and the strain-rate sensitivity of the flow stress. By applying ideas from the theory of the formation of a single adiabatic shear band in torsion, Molinari and Dudzinski (1992) derived the conditions under which continuous chip formation during orthogonal cutting first becomes unstable. More recently, Burns and Davies (1997, 2002) further explained the adiabatic shear localization-induced serrated chip as a bifurcation phenomenon - the limit cycle of the nonlinear dynamic system of tool-chip-workpiece during machining.

The ASBs in serrated chips, which are caused by the instability of the plastic flow of the workpiece material, are usually regularly distributed (Wang et al., 2010). The spacing of these regularly spaced shear bands, i.e., the segment spacing, or the frequency of chip segmentation, is the most important parameter to characterize the chip serration. It affects the fluctuation of the cutting force, temperature rise and even the cutting energy. Thus to predict the segment spacing is quite necessary. There are many classic predictions for the multiple adiabatic shear bands that formed from simlpe shear (Grady and Kipp, 1987; Wright and Ockendon, 1996; Molinari, 1997; Batra and Wei, 2006), however, only few theory works have yet to be presented to predict the segment spacing that occurs during the serrated chip formation processes. Huang and Aifantis (1997) and Huang et al. (2007) proposed a method for thermo-viscoplastic instability in chip formation. The perturbation analysis was carried out to establish the relations for the segment spacing which was obtained by multiplying the chip flow velocity by the characteristic time for instability. Molinari et al. (2002) analyzed the segment spacing in terms of the cutting velocity based on the work of Wright and Ockendon (1996) and Molinari (1997). In their work, the segment spacing was proposed to be related to the characteristic perturbation wave length for which the corresponding perturbation growth rate took a peak maximum value. These fantastic pioneer works give important clues to study the segment spacing by analyzing the shear bands plastic deformation within the PSZ.

During the serrated chip formation, after the thermo-plastic instability takes place, the shear band forms inside the PSZ, and it could evolve to a certain degree before it is taken away from the PSZ by the chip flow. Just because of the evolution of 
the shear band, the shear front surface becomes visible, which makes the chip formation appearing to be serrated. The cutting speed affects the competition between the heat generation and heat diffusion inside the PSZ, thus the evolution degree of shear band could vary with the cutting speed. As has been point out by Meyers et al. (2001), Xue et al. (2002, 2004) and Zhou et al. (2006), the shear band spacing is largely related to the evolution degree of shear bands. Thus the shear band evolution could be a key role in the prediction of segment spacing, which has not been considered yet in the existing models. Moreover, the serrated chip formation in machining involves a highly nonlinear thermo-viscoplastic flow of material. And especially during high speed cutting, some factors, such as momentum diffusion and especially the material convection induced by the rapid chip flow, could become significant (Burns and Davies, 2002; Jiang and Dai, 2009b; Ye et al., 2012). These factors could affect the energy dissipation during shear band evolution, which makes the problem more complicated, and hence a new proper model is necessary to be developed for segment spacing prediction during high speed cutting. In the present work, based on the experimental observations, we developed a new model to describe the evolution process of shear band during the serrated chip formation. The prediction for the segment spacing was further proposed based on the momentum diffusion principle, which was validated by both the orthogonal machining experiments and finite element model (FEM) simulations.

\section{Experimental}

An experimental setup, originally developed in Ye et al. (2012) was used to achieve high speed machining. The device is developed based on the light-gas gun loading technology. It allows real orthogonal cutting under quasi-stationary conditions. The details of this experimental device can be found in the reference of Ye et al. (2012). By using this device, high speed machining of Ti-6Al-4V alloy was undertaken using an uncoated P10 carbide tool, with velocities ranging from $5 \mathrm{~m} / \mathrm{s}$ to $31.2 \mathrm{~m} / \mathrm{s}$. Moreover, to complete the range of cutting speeds, as did by Sutter and Molinari (2005), similar tests were made on a CK6150B computer-controlled lathe with the same tools for cutting speeds going from 0.05 to $5 \mathrm{~m} / \mathrm{s}$. The other test conditions both for higher and lower speed cutting are listed in Table 1. New cutting inserts were used for each test to limit the influence of tool wear. The workpiece material Ti-6Al-4V is a two-phase $(\alpha+\beta)$ titanium alloy, composed of equiaxed Ti $\alpha$ grins surrounded by Ti $\beta$ grains, see Fig. 1. This Ti-6Al-4V alloy has a hardness of HV 410 and a static strength about $850 \mathrm{MPa}$. It must be pointed out that, the upper cutting speed $31.2 \mathrm{~m} / \mathrm{s}$ achieved in this work is extremely high for Ti6Al-4V alloy. According to Schulz and Moriwaki (1992), the lower and upper limit for high-speed machining of titanium alloy is about $1.5 \mathrm{~m} / \mathrm{s}$ and $15 \mathrm{~m} / \mathrm{s}$, respectively.

After cutting, the chips have been collected and embedded into resin. The lateral section was mechanically polished and then etched. Then, the optical microscope and high-resolution scanning electron microscopy (HRSEM, FEI Sirion, spatial resolution $1.5 \mathrm{~nm}$ ) were used to examine the chip morphology.

\section{Results}

As for the lower speed cutting $\left(V_{c}<0.5 \mathrm{~m} / \mathrm{s}\right)$, the chips are continuous, see Fig. 2a. The crystal particles in the continuous chip have undergone a relatively homogenous shear deformation as the grains exhibit a markedly preferred orientation. When it comes to higher speed cutting $\left(V_{c}>0.5 \mathrm{~m} / \mathrm{s}\right)$, the chips are serrated, see Fig. $2 c$ and d. The adiabatic shear bands are widely observed between the saw-teeth, and the degree of segmentation increases as the cutting speed increases. A magnified figure marked in Fig. 2c gives the detailed structures of the shear band. In this figure, several features should be noticed. First, the absence of any cracks or voids within or near the shear bands indicates that the serrated chip formation does not result from the mechanism of periodic crack. Second, the microstructure outside the shear band is quite different from that inside: the grains inside the shear bands are elongated along the shear direction and have undergone an extremely high deformation, while those outside the shear bands remain almost undeformed. In addition, the deformation inside the shear band is not uniform. The grains in the center of the shear band deform more severely than those near the boundary.

Moreover, the structures of the free surface of chip for different cutting speeds were further investigated. Examination of the free surface of the continuous chip reveals a relatively fine distributed lamellar structure (Barry and Byrne, 2002; Wang et al., 2011), see Fig. 2e. For continuous chips, the shear surfaces produced by the shear fronts are smooth, which means the shear band between each adjacent lamella evolves weakly. As for the serrated chips, fine shear front-lamellar structures are evident, see Fig. $2 \mathrm{f}-\mathrm{h}$. The shear surfaces of each adjacent lamella are obviously visible. Furthermore, carefully observation shows that, each primary lamella consists of several distinct or obscure secondary lamellae. During the process of continuous chip formation, the growth of the shear bands is relatively stable, thus all the shear bands evolve to almost a same level (a very low level). However, when it comes to the serrated chip formation, the growth of the shear bands is unstable. As the deformation evolves, some of them grow faster, suppressing the others, and finally form the primary shear bands, see

Table 1

Cutting conditions.

\begin{tabular}{lllll}
\hline Rake angle $\omega$ & Clearance angle $\lambda$ & Uncut chip thickness $b$ & Cutting width $w$ & Cutting speed $V_{c}$ \\
\hline $0^{\circ}$ & $7^{\circ}$ & $0.1 \mathrm{~mm}$ & $2 \mathrm{~mm}$ & $0.05-31.2 \mathrm{~m} / \mathrm{s}$ \\
\hline
\end{tabular}




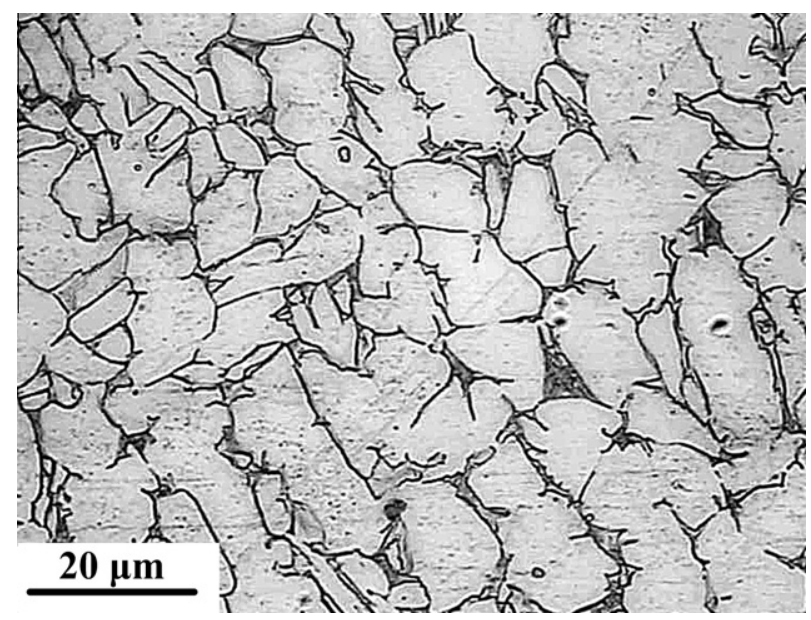

Fig. 1. Microstructure of the as-received material.

Fig. $2 \mathrm{f}$ and $\mathrm{g}$. And the other shear bands which are suppressed by the primary shear bands stop growing, forming the secondary shear bands. Thus, it seems like that the shear band spacing (segment spacing) increases as the shear band evolves, which is agree with the theory of Meyers et al. (2001), Xue et al. (2002, 2004) and Zhou et al. (2006). In addition, it also can be noticed from Fig. 2e-h that, increasing the cutting speed promotes the evolution of the primary shear bands, which makes the shear front surfaces more remarkable, implying a higher degree of segmentation. This tendency is in coincidence with that of the chip formation as shown in Fig. $2 a-d$.

\section{Theoretical modeling and discussion}

\subsection{The momentum diffusion-based shear band evolution model}

As for the continuous chip formation, the cutting tool in relation to the workpiece is schematically presented in Fig. 3a. The deformation of the material ahead of the tool occurs within the PSZ of a finite thickness $h$. The PSZ is assumed to be parallel-sided according to Oxley (1989). The lower boundary $C D$ and the upper boundary EF are parallel and equidistant from the nominal shear plane $A B$. A Lagrangian coordinate system $(x, y)$ is attached to the PSZ. Coordinates $x$ and $y$ are parallel and normal to the direction of shear flow, respectively. The velocity along $x$ direction $\left(V_{x}\right)$ is assumed to change from zero on the lower boundary to $V_{s}$ (chip velocity along the shear direction) on the upper boundary linearly with no discontinuities, as shown in Fig. $3 \mathrm{~b}$. Thus the shear strain rate is homogenous throughout the PSZ. The average shear strain rate $\dot{\gamma}_{0}$ within PSZ is related to the cutting speed $V_{c}$, tool rake angle $\omega$ and shear angle $\varphi$ by (Oxley, 1989).

$$
\dot{\gamma}_{0}=\frac{V_{c} \cos \omega}{h \cos (\varphi-\omega)}
$$

In current analysis, the material is assumed to be rigid-ideal plastic for simplicity, and the homogenous deformation condition of continuous chip is taken as the initial condition for the serrated chip formation. At the initial time $t=0$, the material in the PSZ is undergoing uniform shear at flow stress $\tau_{y}$. The initial velocity field is given by

$$
V_{x}(y)=\dot{\gamma}_{0} y
$$

As the cutting speed increases, the chip formation changes from continuous to serrated, and a primary shear band forms along the shear plane $A B$, as shown in Fig. 3c. After the primary shear band forms, the shear-banding proceeds as the tool keeps on loading. Fig. 3d illustrates the evolution process of the primary shear band. In order to render the theoretical derivation tractable, as did by Grady and Kipp (1987), the shear band is assumed to have a constant thickness $a$, which is much smaller compared with the shear zone thickness. Thus the details of the shear band can be collapsed to a boundary displacement condition at $y=h / 2$. After the shear band forms, large deformation occurs inside the shear band as the localized shear deformation proceeds. Subsequently, thermal softening takes place due to temperature rise and hence the stress relaxes within the shear band. The stress release further propagates outward into the undisturbed media, giving rise to momentum diffusion, which results in two rigid regions between the shear band and the plastic deformation regions, as shown in Fig. 3d. The two rigid regions are assumed to have a same width $\xi$. The rigid-plastic interface propagates outwards as the shear band evolves. It is worth noticing that, the undeformed materials are widely observed between the shear bands in the serrated chips, see Fig. 2c. It seems that these undeformed materials are in rigid regions that created by the momentum diffusion. 


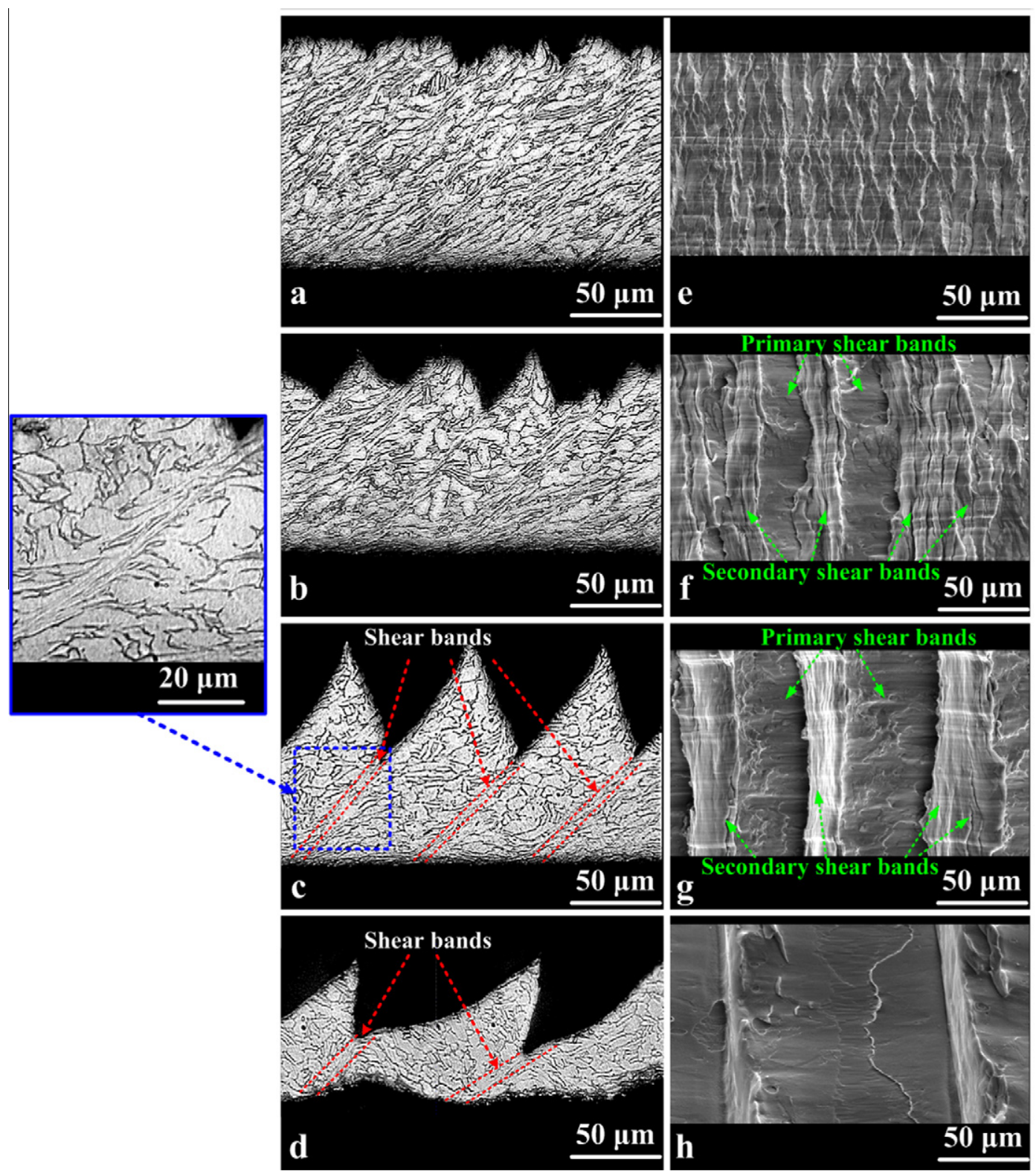

Fig. 2. Microstructure of (a-d) chips obtained at a cutting speed of $0.05,0.5,5$ and $31.2 \mathrm{~m} / \mathrm{s}$ respectively; (e-h) free surfaces of chips obtained at cutting speed of $0.05,0.5,5$, and $31.2 \mathrm{~m} / \mathrm{s}$, respectively.

Moreover, the deformation gradient in the shear band (see the magnified figure in Fig. 2c) also seems like a trace that left by the stress release propagating outward from the shear band center. Both the undeformed material between the shear bands and the deformation gradient in the shear band confirm the momentum diffusion mechanism.

During the shear band evolution, the velocity of the rigid region is controlled by the momentum diffusion. The velocity field at later times, $t>0$, as the rigid-plastic interface propagates to the position $\xi(t)$, is given by (see Fig. $3 \mathrm{~d}$ ).

$$
V_{x}(y, t)=\left\{\begin{array}{lll}
\dot{\gamma}_{0} y & (0 \leqslant y \leqslant h / 2-\xi(t)) \quad(3 \mathrm{a}) \\
\dot{\gamma}_{0}(h / 2-\xi(t)) & (h / 2-\xi(t) \leqslant y \leqslant h / 2) \quad(3 \mathrm{~b}) \\
\dot{\gamma}_{0}(h / 2+\xi(t)) & (h / 2 \leqslant y \leqslant h / 2+\xi(t)) \quad(3 \mathrm{c}) \\
\dot{\gamma}_{0} y & (h / 2+\xi(t) \leqslant y \leqslant h) \quad(3 \mathrm{~d})
\end{array}\right.
$$



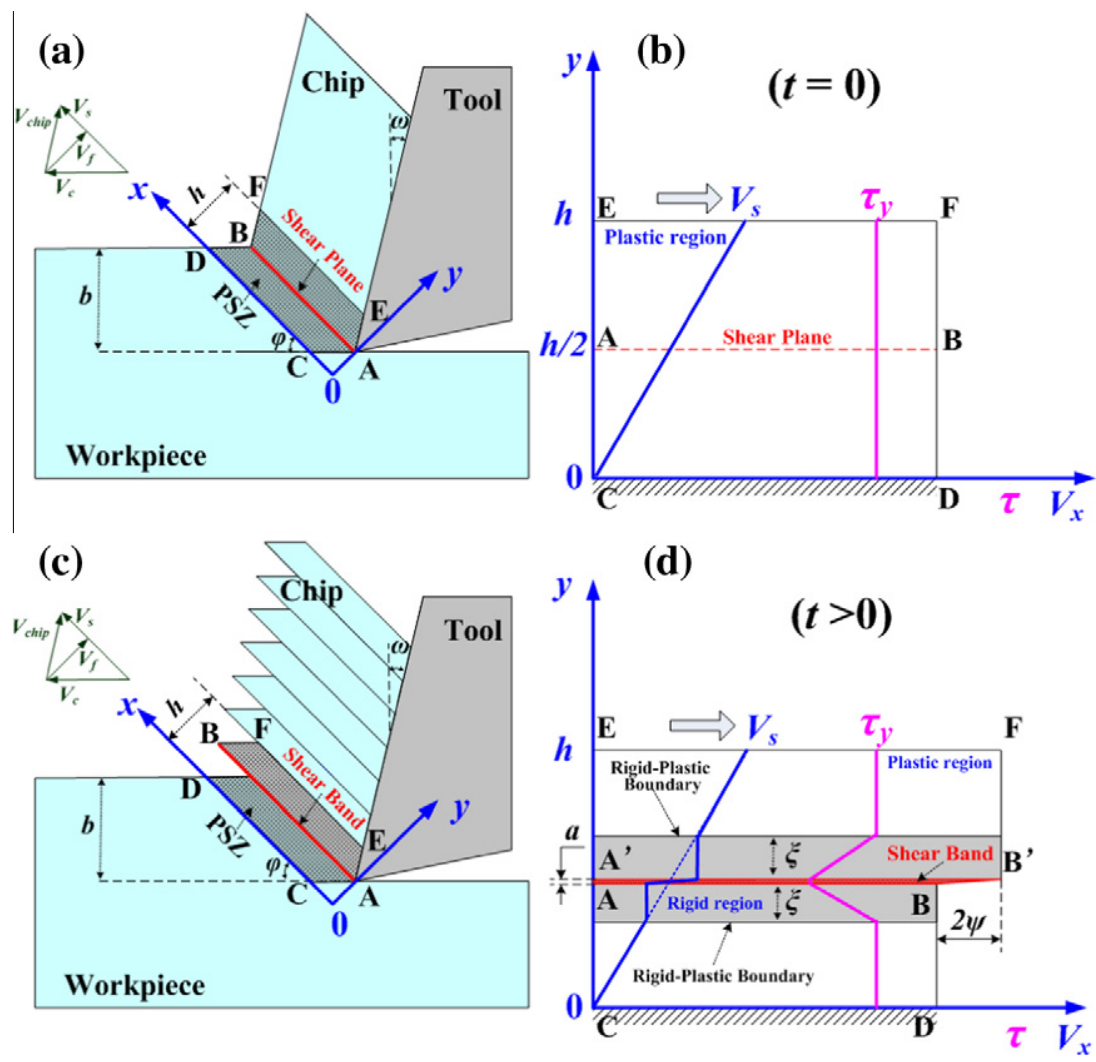

Fig. 3. Cutting model and the velocity and stress field in PSZ: (a,b) for continuous chips and (c,d) for serrated chips.

First, we investigate the momentum diffusion and thermal diffusion for the lower half region of the PSZ $(0 \leqslant y \leqslant h / 2)$. In this region, the change of momentum per unit area during shear band evolution can be obtained from Eqs. (2), (3a) and (3b), and thus the momentum equation can be obtained as:

$$
\rho \dot{\gamma}_{0} \xi \frac{d \xi}{d t}=\tau_{y}-\tau
$$

where $\rho$ is mass density, $\tau_{y}$ the constant shear stress within the plastic region (see Fig. 3d), and $\tau$ is the relaxed shear stress inside the shear band, which is related to the shear band displacement $\psi$ (see Fig. 3d) obeying a softening law.

It should be noticed that, the relative shear band slip is caused by the discontinuity of velocity at $y=h / 2$ (see Eq. (3)), thus the boundary displacement $\psi$ at $y=h / 2$ satisfies

$$
\frac{d \psi}{d t}=\frac{1}{2} \dot{\gamma}_{0}\left[\left(\frac{h}{2}+\xi\right)-\left(\frac{h}{2}-\xi\right)\right]=\dot{\gamma}_{0} \xi
$$

As the shear band evolves, the boundary displacement $\psi$ increases and the shear stress $\tau$ in the shear band decreases. When the shear stress in the shear band vanishes as the shear displacement reaches a critical $\psi_{c}$, the shear band is regarded as being fully mature. However, during cutting, the chip flow always takes materials away from the PSZ. Thus, there is no sufficient time for the shear band to fully develop inside the PSZ. In addition, as has been discussed in Section 3, the actual evolution degree of the shear band has a significant influence on the shear band spacing, and is not constant but varies with the cutting speed. In consideration of these, we further define a parameter $X$ to describe the degree of shear band evolution,

$$
X=\frac{\psi_{a}}{\psi_{c}} \quad(0 \leqslant X \leqslant 1)
$$

where $\psi_{a}$ is the actual boundary displacement during serrated chip formation. When $X=1$, the shear band is fully mature, and the stress in the shear band falls to zero. And $X=0$ demonstrates that the shear band has just formed without evolution. Moreover, a linear relaxation of shear stress is used to approximately describe the relation of unloading stress,

$$
\tau=\tau_{y}\left(1-X \frac{\psi}{\psi_{a}}\right)
$$


Combining Eqs. (4), (5), and (7) with their initial condition $\psi(0)=\xi(0)=0$, leads to a solution for the boundary displacement

$$
\psi=X \frac{\tau_{y} \dot{\gamma}_{0}}{18 \rho \psi_{a}} t^{3}
$$

and rigid-plastic interface position

$$
\xi=X \frac{\tau_{y}}{6 \rho \psi_{a}} t^{2}
$$

When $\psi=\psi_{a}$, the actual shear-band evolution time $t_{a}$ and the actual width of the rigid region $\xi_{a}$ is obtained, that is

$$
\begin{aligned}
& t_{a}=\left(\frac{18 \rho \psi_{a}^{2}}{X \tau_{y} \dot{\gamma}_{0}}\right)^{1 / 3} \\
& \xi_{a}=\left(\frac{3 X \tau_{y} \psi_{a}}{2 \rho \dot{\gamma}_{0}^{2}}\right)^{1 / 3}
\end{aligned}
$$

During the evolution of shear band, the relaxation of shear stress is due to thermal softening of the material. The constitutive law of material usually has the general form

$$
\tau=f(\gamma, \dot{\gamma}, \theta)
$$

where $\gamma$ is the shear strain, $\dot{\gamma}$ shear strain rate and $\theta$ the temperature rise.

Shear banding deals with complicated constitutive behaviors of materials such as dislocation, strain gradient effect, size effect and recrystallization, and some relevant constitutive models have been proposed to describe these behaviors (see the paper of Liang and Khan (1999) and Farrokh and Khan (2009)). However, in the present work, in order to render the analysis tractable, a simplified constitutive law is adopted to describe the coupling effects of rate-hardening and thermal softening during the shear band evolution as did by Grady and Kipp (1987), Grady (1992) and Ikeda et al. (1998), that is

$$
\tau=\tau_{y}\left(\gamma_{0}, \dot{\gamma}_{0}\right)(1-\alpha \theta)+\eta \dot{\gamma}_{\text {rel }}
$$

where $\alpha$ is the thermal softening coefficient, $\eta$ the strain rate hardening coefficient. $\tau_{y}\left(\gamma_{0}, \dot{\gamma}_{0}\right)$ is the initial yield stress in the plastic region before shear localization, which is related to the average shear strain $\gamma_{0}$ and shear strain rate $\dot{\gamma}_{0}$ in the PSZ. $\dot{\gamma}_{\text {rel }}=\frac{2}{a} \frac{d \psi}{d t}$ is the shear strain rate in the shear band caused by the shear band slip.

Through Eqs. (7), (8), and (13), the time dependence of the shear-band temperature rise can be determined from the mechanical solution, given by

$$
\theta=\frac{X^{2} \tau_{y} \dot{\gamma}_{0}}{18 \rho \alpha \psi_{a}^{2}}\left(t^{3}+\frac{6 \eta \psi_{a}}{X a \tau_{y}} t^{2}\right)
$$

During the process of serrated chip formation, the deformation of the PSZ almost concentrated in the shear band. The energy equation governing the thermo-mechanical deformation of the shear band can be written as (Burns and Davies, 1997; Jiang and Dai, 2009b; Grady, 1992)

$$
\frac{d \theta}{d t}=\frac{2 \tau}{\rho c a} \frac{d \psi}{d t}-\frac{2 \lambda}{a^{2}} \theta-\frac{V_{f}}{a} \theta
$$

where $c$ is thermal capacity, $\lambda$ thermal diffusivity, and $V_{f}=V_{c} \sin \varphi$ is the material convection velocity normal to the shear direction. Eq. (15) states that there are three different physical processes that can alter the temperature in the shear band: heat generation due to plastic working (the first term), diffusion and material convection (the second and third terms, respectively). The material convection is due to the chip moving outwards the PSZ, which takes heat away and could be important in high speed machining process (Burns and Davies, 2002; Jiang and Dai, 2009b; Ye et al., 2012).

We now introduce $\Gamma_{a}$ to describe the actual energy dissipated within the shear band as deformation proceeds to the actual displacement $\psi_{a} . \Gamma_{a}$ is the energy dissipated per unit area within one-half of the shear band (Anderson, 2005), identified as

$$
\Gamma_{a}=\frac{2-X}{2} \tau_{y} \psi_{a}
$$

Integrating the energy equation (15) over the actual time $t_{a}$ with Eqs. (7) and (14), and by using Eq. (16) it yields an implicit relation of the actual dissipation energy with the shear band thickness

$$
\frac{2}{X} \bar{\Gamma}_{a} \bar{a}=\bar{a}^{2}+\frac{(1+\bar{a} M) \bar{\Gamma}_{a}^{2 / 3}}{X^{1 / 3}(2-X)^{2 / 3}}+R\left(\frac{\bar{a} \bar{\Gamma}_{a}^{1 / 3}}{X^{2 / 3}(2-X)^{1 / 3}}+\frac{4(1+\bar{a} M)}{3 X(2-X)} \frac{\bar{\Gamma}_{a}}{\bar{a}}\right)
$$


with the variables given as

$$
\bar{a}=\frac{a}{a_{0}}, \quad \bar{\Gamma}_{a}=\frac{\Gamma_{a}}{\Gamma_{0}}, \quad M=\frac{V_{f} a_{0}}{2 \lambda}, \quad R=2 \eta \sqrt{\frac{\dot{\gamma}_{0}}{\rho \lambda \tau_{y}}}
$$

where $a_{0}=\left(\frac{9 \rho^{3} c^{2} \lambda^{3}}{\tau_{y}^{3} \alpha^{2} \gamma_{0}}\right)^{1 / 4}$ and $\Gamma_{0}=\frac{\rho c}{\alpha}\left(\frac{9 \rho^{3} c^{2} \lambda^{3}}{\tau_{y}^{3} \alpha^{2} \hat{\gamma}_{0}}\right)^{1 / 4}$ are the special shear band thickness and energy dissipation without considering the rate-hardening and material convection for a fully mature shear band, respectively. Moreover, it is noticed that, $M$ is a Peclet number that reflects the magnitude of the ratio between material convection and thermal diffusion. As the cutting speed increases, there is less time for the heat conducted into the workpiece/chip, thus the thermal diffusion decreases. Moreover, increasing the cutting speed accelerates the chip flow and hence enhances the material convection. Therefore, the Pelect number $M$ increases with increasing the cutting speed, and $M$ could be significant as the cutting speed is sufficient high. When the material convection is neglected, the Pelect number $M$ vanishes. And the dimensionless parameter $R$ reflects the effect of material rate-hardening, which vanishes when the workpiece is a rate-insensitive material.

It is found that, the shear band always seeks a optimum thickness to minimize the energy dissipated in the shear-band relaxation process (Grady and Kipp, 1987; Grady 1992), thus $\Gamma_{a}$ and $a$ satisfy

$$
\frac{\partial \Gamma_{a}}{\partial a} 0
$$

According to the minimum energy principle (19), another implicit relation of the dissipation energy with the optimum shear band thickness is obtained:

$$
\bar{\Gamma}_{a}=X \bar{a}+\frac{M X^{2 / 3} \bar{\Gamma}_{a}^{2 / 3}}{2(2-X)^{2 / 3}}+R\left(\frac{X^{1 / 3} \bar{\Gamma}_{a}^{1 / 3}}{2(2-X)^{1 / 3}}-\frac{2}{3(2-X)} \frac{\bar{\Gamma}_{a}}{\bar{a}^{2}}\right)
$$

By solving the system of Eqs. (17) and (20), we can get the optimum shear band thickness $a$ and the corresponding dissipation energy $\Gamma_{a}$. Then, the actual rigid-plastic interface position $\xi_{a}$ can be determined from Eqs. (11) and (16).

As for the upper half region of the PSZ $(h / 2 \leqslant y \leqslant h)$, the same momentum equation (4) is obtained. Thus the optimum shear band thickness and dissipation energy for the upper half region $(h / 2 \leqslant \mathrm{y} \leqslant \mathrm{h})$ also have to satisfy the Eqs. (17) and (20). This means that, both for the upper and the lower half regions of PSZ, a same shear band thickness is achieved. So the assumption that the two rigid regions have a same width is reasonable. And thus, the segment spacing for the serrated chip formation can be given by

$$
L=2 \xi_{a}=2\left(\frac{3 X}{(2-X)} \frac{\Gamma_{a}}{\rho \dot{\gamma}_{0}^{2}}\right)^{1 / 3}
$$

When the workpiece material is rate-insensitive, the dimensionless parameter $R$ in Eqs. (17) and (20) vanishes. This leads to

$$
L=2\left(\frac{9 \rho c^{2} k}{\tau_{y} \alpha^{2} \dot{\gamma}_{0}^{3}}\right)^{1 / 4}\left[\frac{X^{2}}{2-X} \bar{a}\left(1+\frac{M}{2} \bar{a}\right)\right]^{1 / 3}
$$

where $\bar{a}=\frac{X^{1 / 2}}{2-X}\left(\sqrt{\frac{2-X}{X^{1 / 2}}+\frac{M^{2}}{16}}+\frac{M}{4}\right)$. It should be noticed that, at lower cutting speed, the chip flow is very slow, and the effect of material convection is usually negligible, thus the Peclet number $M$ vanishes in Eq. (22). Actually, at lower cutting speed, the evolution of shear band is weak, thus $(2-X)$ is often much bigger than $X^{1 / 2}$. Moreover, under the cutting conditions described in Section $1, M^{2} \ll 16\left(M^{2}<1.6\right)$ holds when the cutting speed is lower than $60 \mathrm{~m} / \mathrm{s}$. So, when the cutting speed is not especially high, it is true that $(2-X) / X^{1 / 2} \gg M^{2} / 16$, thus the Eq. (22) can be further reduced to

$$
L=2 \frac{X^{3 / 4}}{(2-X)^{1 / 2}}\left(\frac{9 \rho c^{2} k}{\tau_{y} \alpha^{2} \dot{\gamma}_{0}^{3}}\right)^{1 / 4}
$$

When the shear band is fully mature, i.e., $X=1$, Eq. (23) reduces to the model of Grady and Kipp (1987). It can be seen that, at lower cutting speed, the segment spacing is dominated not only by the strain rate loaded on the PSZ but also by the evolution degree of shear band. According to Eq. (23), when the evolution degree of the shear band is not considered, the segment spacing decreases quickly with the cutting speed approximately as $V_{c}^{-3 / 4}$, which is same as the models of Wright and Ockendon (1996), Molinari (1997) and Grady and Kipp (1987). However, increasing the cutting speed promotes the evolution of shear band, which retards the reduction of segment spacing, and hence a smoother slope is obtained in the log-log diagram of the segment spacing versus cutting speed.

As has been discussed above, increasing the cutting speed enlarges the Pelect number $M$. Undoubtedly, when the cutting speed is sufficient high, the material convection could become dominant, and the Peclet number $M$ could be much higher than $4(2-X)^{1 / 2} / X^{1 / 4}$, thus Eq. (22) can be rewritten as 


$$
L=\frac{3 X}{2(2-X)} \frac{\rho c h}{\tau_{y} \alpha} \frac{\sin \varphi \cos (\varphi-\omega)}{\cos \omega}
$$

Thus at especially higher cutting speed, the segment spacing tends to be mainly dominated by the evolution degree of shear band.

\subsection{Comparison with the experimental results}

For the continuous chip, according to Merchant (1945), the relation between cutting force $F_{c}$ and shear stress $\tau$ in the shear plane (shear band for the serrated chip formation) can be obtained by treating the chip as a 'separate' body in stable mechanical equilibrium, that is

$$
F_{c}=\tau \cdot b w \frac{\cos (\beta-\omega)}{\cos (\varphi+\beta-\omega) \sin \varphi}
$$

where $b$ is the uncut chip thickness, $w$ the cutting width, $\beta$ the tool-chip friction angle. It can be seen from Eq. (25a) that the cutting force is approximately in proportion to the shear stress in the shear band.

Before the shear band evolves, the shear stress in the shear band equals to $\tau_{\mathrm{y}}$ that corresponds to the maximum cutting force, $F_{\max }$, that is

$$
F_{\max }=\tau_{y} \cdot b w \frac{\cos (\beta-\omega)}{\cos (\varphi+\beta-\omega) \sin \varphi}
$$

As the shear band evolves, the shear stress unloads, and thus the cutting force decreases. When the shear band evolves to the actual degree $X$, the actual shear stress $\tau_{a}$ in the shear band can be obtained according to Eq. (7)

$$
\tau_{a}=\tau_{y}\left(1-X \frac{\psi_{a}}{\psi_{a}}\right)=\tau_{y}(1-X)
$$

At this time, the corresponding cutting force is minimum $\left(F_{\min }\right)$, that is

$$
F_{\min }=\tau_{a} \cdot b w \frac{\cos (\beta-\omega)}{\cos (\varphi+\beta-\omega) \sin \varphi}
$$

Thus, the actual evolution degree of the shear band can be worked out from the fluctuation of the cutting force by using Eqs. (25b)-(25d), that is

$$
X=1-F_{\min } / F_{\max }
$$

To obtain the evolution of the cutting force and further validate the theoretical model, FEM was developed to simulate the cutting processes. The FEM is a fully coupled thermal-mechanical model, developed in the commercially available software, ABAQUS/Explicit. The tool was treated here for simplicity as an analytical rigid body. To model the thermal-viscoplastic behavior of the workpiece material, the Johnson-Cook $(\mathrm{J}-\mathrm{C})$ law was used, which can be represented by the following formula:

$$
\tau=\left(A+B\left(\frac{\gamma}{\sqrt{3}}\right)^{n}\right)\left[1+C \ln \left(\frac{\dot{\gamma}}{\dot{\gamma}_{\text {ref }}}\right)\right]\left[1-\left(\frac{\theta}{\theta_{\text {melt }}}\right)^{m}\right]
$$

where the Johnson-Cook parameter $A, B, C, n, m, \theta_{\text {melt }}$ and $\dot{\gamma}_{\text {ref }}$ are defined in Table 2.

The Johnson-Cook parameter values and other mechanical properties used to describe the behavior of Ti-6Al-4V workpiece are also specified in Table 2 (Lee and Lin, 1998). It should be pointed out that, during cutting, the workpiece materials in the PSZ undergo large strains, and 'strain-hardening saturation' may occur for some materials (Childs et al., 2000). Arrazola

Table 2

Mechanical properties and parameters for Ti-6Al-4V.

\begin{tabular}{lll}
\hline Properties and parameters & Notation & Value \\
\hline Density & $\rho$ & $4430 \mathrm{~kg} \mathrm{~m}^{-3}$ \\
Thermal diffusivity & $\lambda$ & $2.95 \mathrm{~m}^{2} \mathrm{~s}^{-1}$ \\
Specific heat & $c$ & $520 \mathrm{~J} \mathrm{~kg}^{-1} \mathrm{~K}^{-1}$ \\
Initial yield stress for J-C model & $A$ & $452 \mathrm{MPa}$ \\
Hardening modulus for J-C model & $B$ & $287 \mathrm{MPa}$ \\
Strain rate dependency coefficient for J-C model & $C$ & 0.028 \\
Work-hardening exponent for J-C model & $n$ & 0.28 \\
Thermal softening exponent for J-C model & $m$ & 1 \\
The reference stain rate for J-C model & $\dot{\gamma}_{\text {ref }}$ & $1.7325 \times 10^{-5} \mathrm{~s}^{-1}$ \\
Melting temperature rise for J-C model & $\theta_{\text {melt }}$ & $1580 \mathrm{~K}$ \\
Thermal softening coefficient for Eq. (12) & $\alpha$ & $6.5 \times 10^{-4} \mathrm{~K}^{-1}$ \\
Strain rate hardening coefficient for Eq. (12) & $\eta$ & $0.75 \mathrm{~Pa} \mathrm{~s}^{-1}$
\end{tabular}



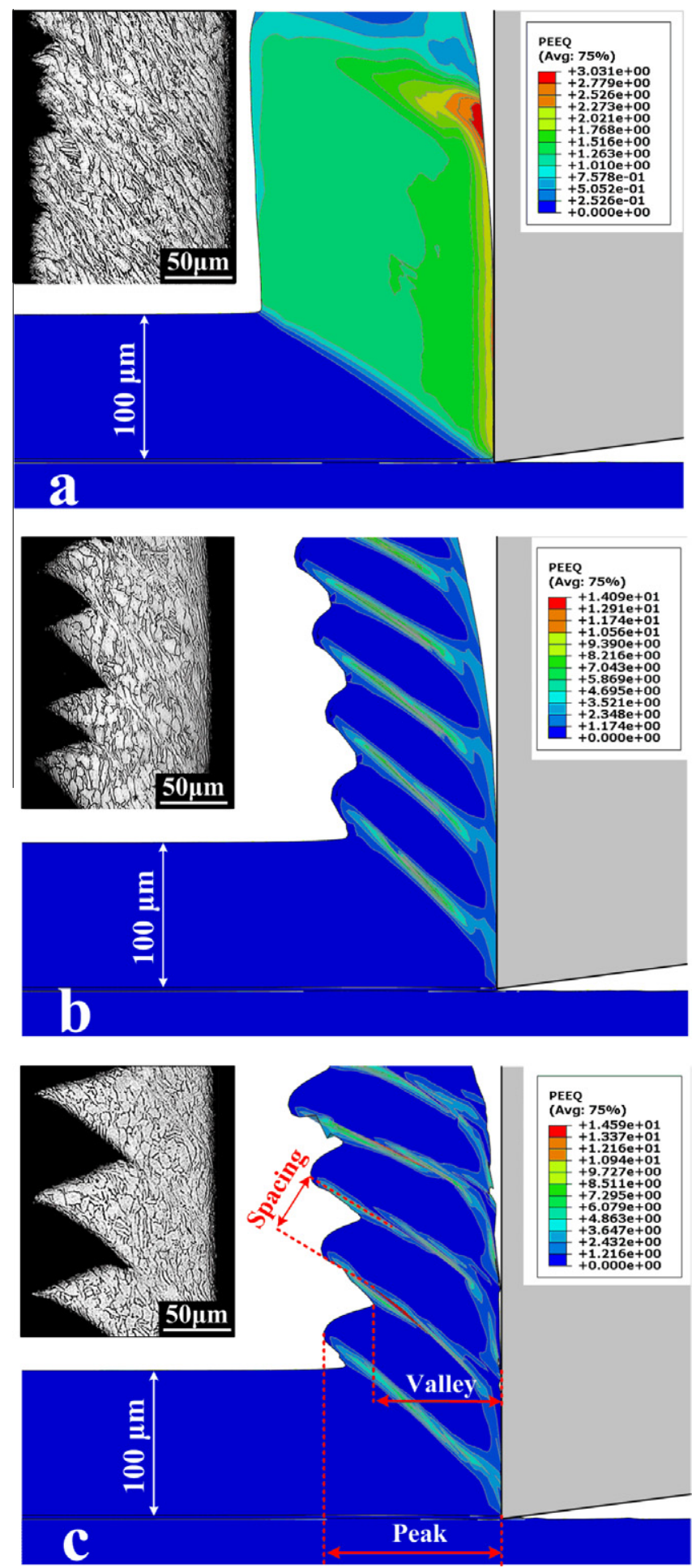

Fig. 4. Simulated chip morphology (a) $V_{c}=0.05 \mathrm{~m} / \mathrm{s}$; (b) $V_{c}=1 \mathrm{~m} / \mathrm{s}$ and (c) $V_{c}=5 \mathrm{~m} / \mathrm{s}$.

et al. $(2007,2010)$ have pointed out that the tendency to create serrated chip decreases when the strain-hardening coefficient is raised. Thus 'strain-hardening saturation' may influence the serrated chip prediction. However, in this work, the 'strain-hardening saturation' effect was not considered. And the more exactly simulation will be carried out in our later work to consider the effect of 'strain-hardening saturation'. 
The extended Coulomb friction model is chosen in this work to model the tool-chip interaction, and the friction coefficient is set to be 0.3 as a constant (Calamaz et al., 2008). The separation of the chip from the workpiece is modeled with the help of a failure zone (Hortig and Svendsen, 2007). Up to failure, this zone behaves according to the J-C-based model described in Eq. (27). The failure of this zone takes place at a critical value $\gamma_{p}$ of the accumulated equivalent inelastic deformation, and hence the chip separates from the surrounding workpiece in a defined distance from the tool tip. In this work, the $\gamma_{p}$ is set to a value of 2.0 (Calamaz et al., 2008). The separation criteria could lead to additional energy for new surface formation (Atkins, 2003, 2009). However, in the present work, the failure zone $(5 \mu \mathrm{m})$ is much thinner than the uncut chip thickness, and hence the energy for the new surface formation can be neglected. The cutting parameters listed in Table 1 were used during the FEM simulations.

The simulated chip morphologies for different cutting speeds are shown in Fig. 4. The serrated chip morphology can be characterized by three parameters, i.e., segment spacing, valley and peak, see Fig. 4c. For all simulations, the shear angle is about $45^{\circ}$, thus $\varphi=45^{\circ}$ is adopted for the subsequent calculations. The comparison of simulated and experimental chip morphology is presented in Fig. 5. It can be seen that the dimensions of simulated results agree well with the experimental ones. The good agreements between the simulations and experiments indicates the FEM simulations employed in this work can give accurate predictions for the chip morphologies and also the cutting forces.

Fig. 6 gives the evolutions of the simulated cutting forces with cutting distance for different cutting speeds. At lower speed, the chip is continuous, thus the cutting force is almost constant after the cutting process stabilized. As for higher speed cutting, chip is serrated, and a cyclic cutting force is produced. It can be seen that, with increasing the cutting speed, the perturbation of the cutting force rises rapidly first, and then the increase of cutting force perturbation slows down when the cutting speed is higher than $10 \mathrm{~m} / \mathrm{s}$. This means higher cutting speed renders a higher degree of segmentation. This is in accordance with the experimental result, see Fig. 2. Moreover, based on the evolutions of simulated cutting forces, the evolution degrees of shear bands during serrated chip formation are obtained by using Eq. (26). Moreover, by using Eq. (1), where the thikness of the PSZ $(h)$ is set to be $1 / 10$ of the uncut chip thickness (Burns and Davies, 1997, 2002), the evolution degree of shear bands is plotted with the average shear strain rate that loaded on the PSZ, as shown in Fig. 7. It can be found that, with increasing the strain rate (cutting speed), the evolution degree of shear band first rises rapidly, and then increases gradually with an almost constant slope when the strain rate is higher than $1.5 \times 10^{6} \mathrm{~s}^{-1}$. It is generally known that, increasing the cutting speed increases the strain rate that loaded on the PSZ. This promotes the shear band evolution, leading to a rapid increase of shear band evolution degree. However, when the cutting speed is higher enough, the effect of chip flow becomes remarkable. With increasing the cutting speed the chip flow accelerates rapidly, which reduces the evolution time of the shear band and finally retards the increase of the shear band evolution degree. According to Fig. 7, the relationship between the evolution degree of shear band and the strain rate loaded on the PSZ during machining Ti-6Al-4V can be approximately established, that is

$$
\begin{aligned}
& X=0.160 \times\left(\dot{\gamma}_{0} / 10^{6}\right)^{0.65} \text { for } 0<\dot{\gamma}_{0} \leqslant 1.5 \times 10^{6} \\
& X=0.208+0.03 \times\left(\dot{\gamma}_{0} / 10^{6}-1.5\right) \quad \text { for } \dot{\gamma}_{0} \geqslant 1.5 \times 10^{6}
\end{aligned}
$$

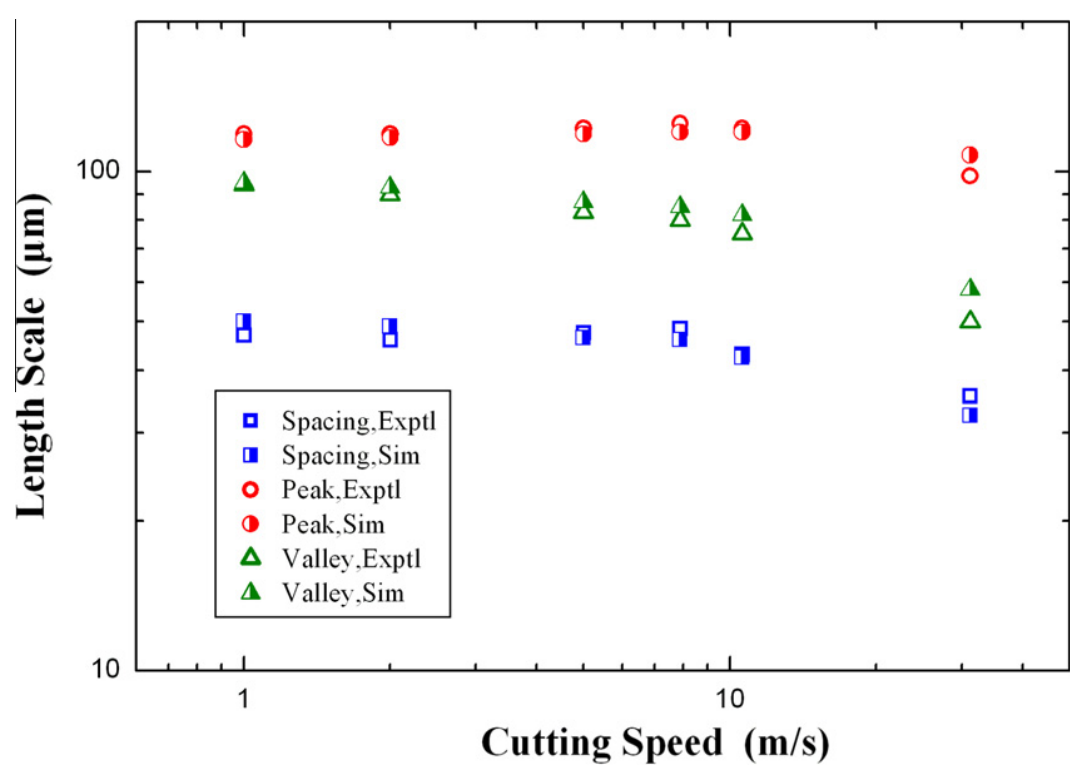

Fig. 5. Comparison of predicted chip dimensions with experimental data. 


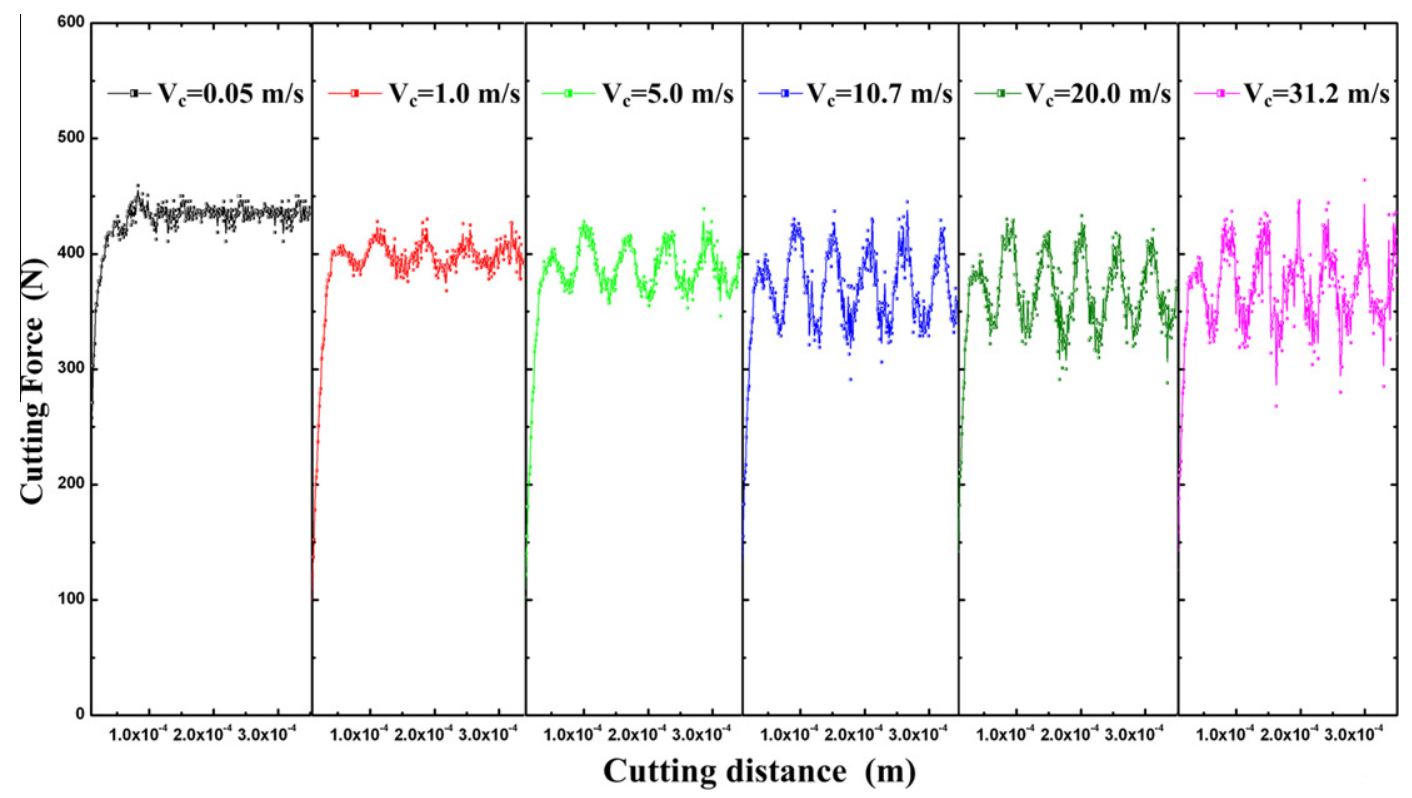

Fig. 6. Cutting force evolves with cutting distance for different cutting speed.

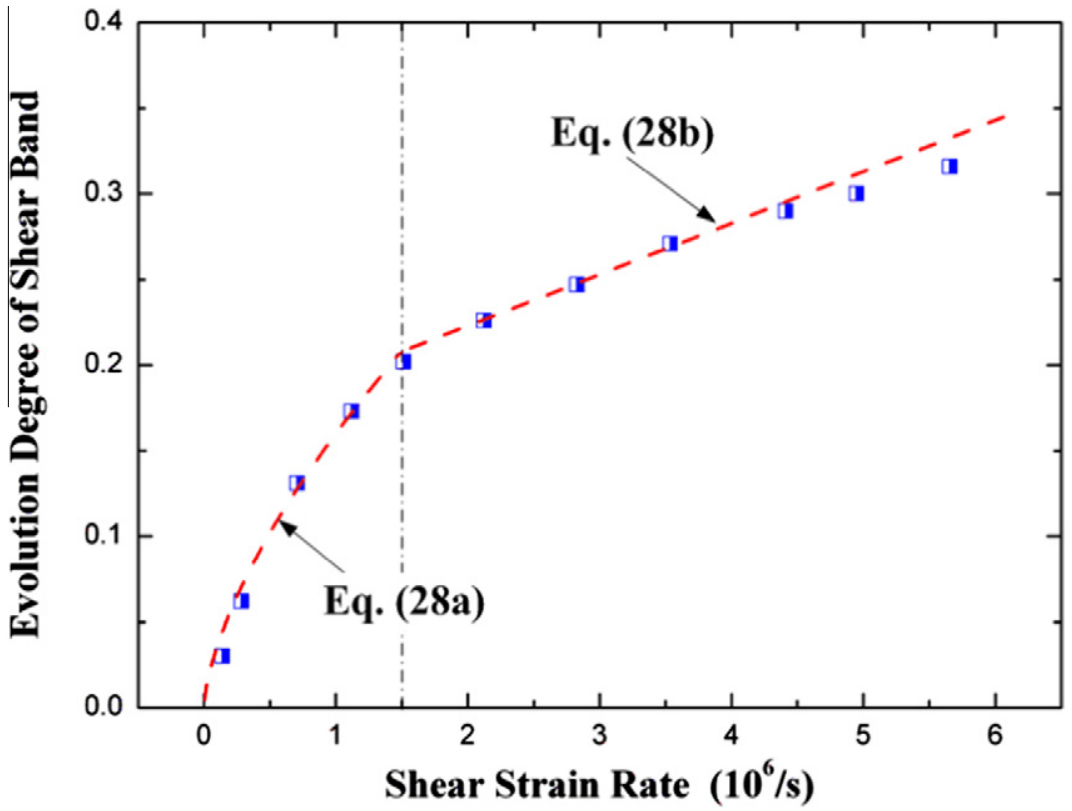

Fig. 7. Evolution degree of shear band versus strain rate loaded on the PSZ

Once the cutting conditions (cutting speed, uncut chip thickness, tool rake angle and shear angle) and the evolution degree of the shear band are known, the predict segment spacing for the theoretical model presented in Section 4.1 can be calculated. Followed the J-C model, the initial yield stress in Eq. (13) is given in the form of

$$
\tau_{y}\left(\gamma_{0}, \dot{\gamma}_{0}\right)=\left(A+B\left(\frac{\gamma_{0}}{\sqrt{3}}\right)^{n}\right)\left[1+C \ln \left(\frac{\dot{\gamma}_{0}}{\dot{\gamma}_{\text {ref }}}\right)\right]
$$

where the values of $A, B, n, C$ and $\dot{\gamma}_{\text {ref }}$ are equal to that of J-C model listed in Table 2. According to Oxley (1989), the average shear strain $\gamma_{0}$ can be given by

$$
\gamma_{0}=\frac{\cos \omega}{2 \sin \varphi \cos (\varphi-\omega)}
$$




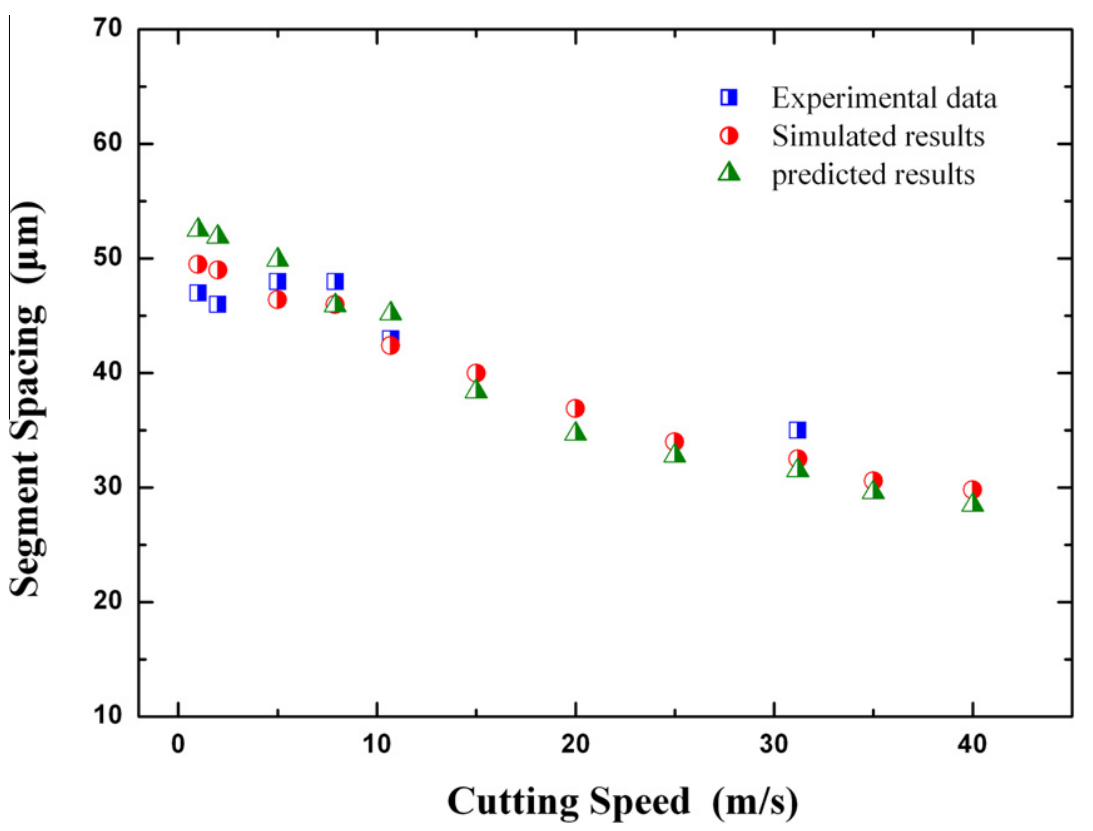

Fig. 8. The predicted, simulated and experimental results for segment spcaing.

Moreover, the thermal softening coefficient $\alpha$ and strain rate hardening coefficient $\eta$ for Eq. (13) are set to be $6.5 \times 10^{-4}\left(\mathrm{~K}^{-1}\right)$ and $0.75\left(\mathrm{~Pa} \mathrm{~s}^{-1}\right)$ respectively to make Eq. (13) describe almost the same material behavior with the J-C model (Eq. (27)).

Fig. 8 gives the predicted segment spacing with the simulated and experimental results. Both for experimental and simulated results, we measured the total spacing of 10 randomly selected adjacent segments, and the average spacing is set to be the segment spacing. It is noticed that the predicted result matches the simulated and experimental data very well, especially at higher cutting speeds. Moreover, by using Eq. (28) to estimate the evolution degree of shear band, we have compared the predicted segment spacing with the pubulised works (Molinari et al., 2002; Cotterell and Byrne, 2008; Amin et al., 2007; Chen et al., 2011; Bäcker, 2006; Sun et al., 2009., all for Ti-6Al-4V alloy), as shown in Fig. 9. The experimental/simulated data for the published works covers wide ranges of cutting speed (0.1 m/s-22 m/s (Molinari et al., 2002)) and uncut chip thickness (0.035 mm (Bäker, 2006)-0.256 mm (Sun et al., 2009)). It can be seen from Fig. 9 that, very good agreements once again achieve between the predicted results and the publised experimental/simulated segment spacing. On the whole, the momentum diffusion-based shear band evolution model can give accurate predictions for the segment spacing over a wide range of cutting speeds.

As for the multiple adiabatic shear bands that formed from simple shear, some theoretical predictions have been proposed to characterize the spatial distribution of shear bands. Based on the analysis of small perturbations, for materials obeying the constitutive relation $\tau=\tau_{y}(1-\alpha \theta) \dot{\gamma}^{\zeta}$, Wright and Ockendon (1996) defined the shear band spacing as $L_{W O}=2 \pi\left(\frac{\zeta^{3} \rho c^{2} k}{\tau_{0} \alpha^{2} \gamma_{0}^{3}}\right)^{1 / 4}$ (WO model). And the expression of Molinari (1997) is $L_{M}=2 \pi\left(\frac{\zeta^{3} \rho c^{2} k\left(1-a \theta_{0}\right)^{2}}{(1+\zeta) \tau_{0} \alpha^{2} \gamma_{0}^{3}}\right)^{1 / 4}$, here $\zeta$ is the strainrate-hardening exponent, and $\theta_{0}$ and $\tau_{0}=\tau_{y}\left(1-\alpha \theta_{0}\right) \dot{\gamma}_{0}^{\zeta}$ are the temperature rise and shear stress inside the shear band just when the shear band forms. As for the rate-insensitive materials obeying the constitutive relation $\tau=\tau_{y}(1-\alpha \theta)$, Grady and Kipp (1987) (GK model) obtained the spacing for the fully mature shear bands $(X=1)$ by analyzing the shear band evolution based on the momentum diffusion principle, that is $L_{G K}=2\left(\frac{9 \rho c^{2} k}{\tau_{y} \alpha^{2} \gamma_{0}^{3}}\right)^{1 / 4}$.

In Fig. 10 the predicted segment spacing (Eq. (21)) for the proposed model is compared with the predictions for WO, Molinari and GK models. It can be seen that the present predicted model agrees well with the experimental and simulated results from low to high cutting speeds. It is worth noticing that, the WO/Molinari models are based on the perturbation analysis, they give a good explain of the initial growth of shear band at an early stage. While the GK model is based on studying the momentum diffusion for the sufficiently developed shear band. It describes well the later stage of the shear localization. Since the evolution of the shear bands enlarges their spacing (Meyers et al., 2001; Xue et al., 2002, 2004; Zhou et al., 2006), the predictions of the GK model is always larger that those of WO/Molinari models. It seems like that, the WO/Molinari and GK models give the lower bound and higher bound of the shear band spacing, respectively. As for machining, at lower speeds, the shear bands are weakly developed, thus the WO/Molinari models can give good pridictions for the segment spacing. However, as for higher speed cutting, especially at extremely high cutting speeds, the shear bands could evolve to a certain high level during the process of serrated chip formation. Thus the predictions for the WO/Molinari models become 


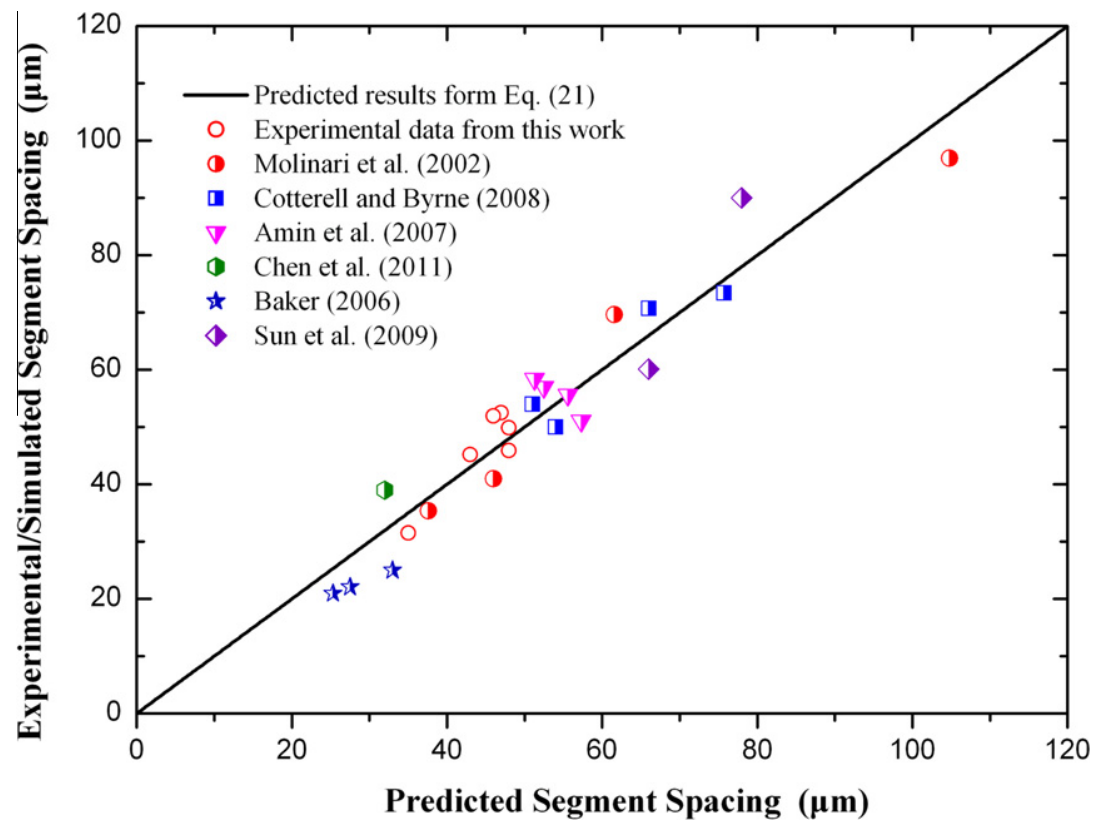

Fig. 9. Correlation between predicted and experimental/simulated segment spacing for published works.

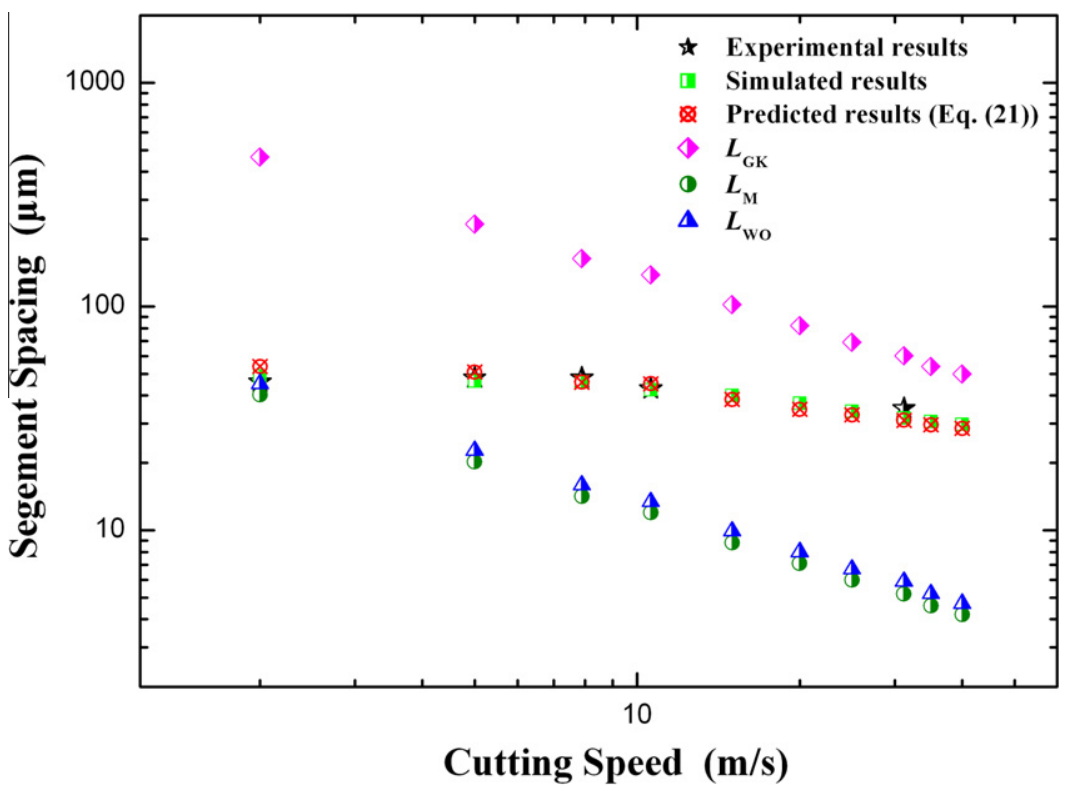

Fig. 10. Predicted segment spacing for different models. Here the strain-rate-hardening exponent $\zeta$ is set to be 0.017 (Xue et al., 2002 ), $\theta_{o}$ and $\tau_{o}$ are approximately taken as the average temperature rise and average flow shear stress in the PSZ to compute $L_{W o}$ and $L_{M}$.

much more smaller, and the GK model trends to give more resonable predictions. As for the present predicted model, it takes into account both the effect of evolution degree of shear band on the segment spacing and the correlation between the shear band evolution degree and cutting speed. Thus the present shear band evolution model can give good predictions for the segment spacing of the serrated chips over a wide range of cutting speeds.

In addition, in order to investigate the influence of rate-hardening and material convection on the segment spacing, three typical cases were considered: (i) with considering the effects of both rate-hardening and material convection (Eq. (21)); (ii) without considering the effect of rate-hardening only (Eq. (22)); (iii) without considering the effects of both rate-hardening and material convection (Eq. (23)). The predicted segment spacing for these three cases was given in Fig. 11. It can be seen that, the predicted segment spacing for case (ii) is a little bit bigger than that for case (iii), and when the effect of ratehardening is further considered (case i), a much more larger segment spacing is achieved. This trend demonstrates that both 


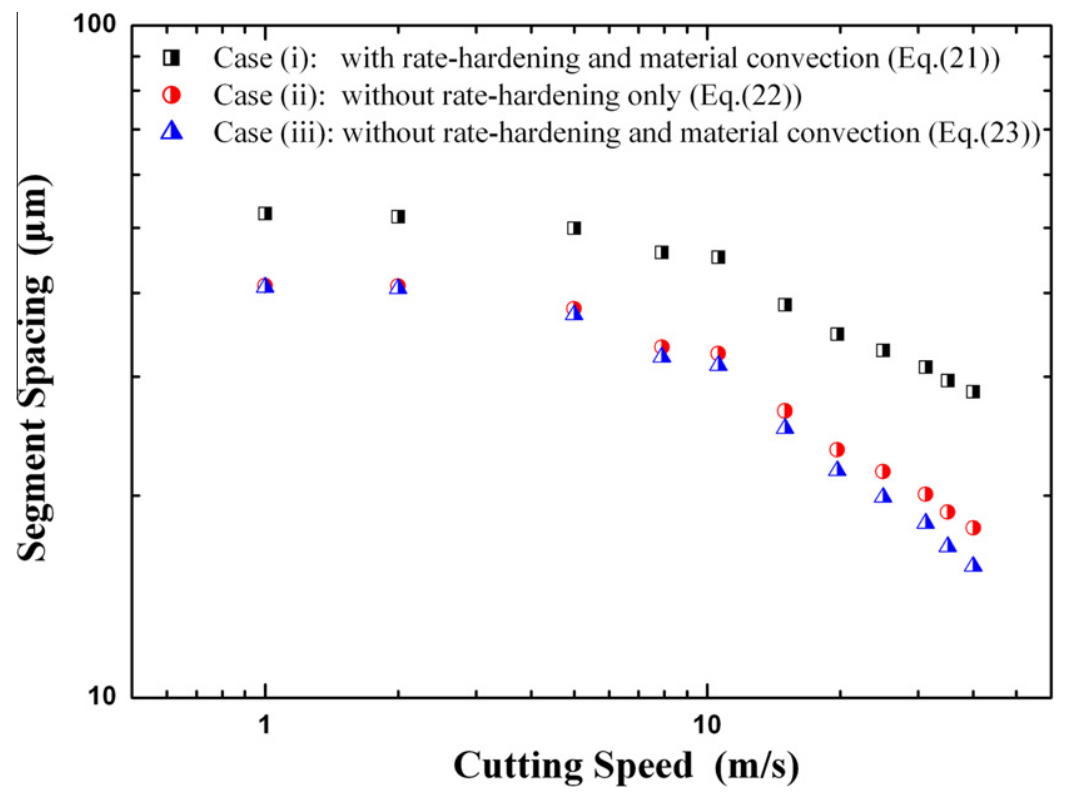

Fig. 11. Three typical predicted segment spacing for Ti-6Al-4V.

the rate-hardening and material convection enlarge the segment spacing. It should be noticed that, the rate-hardening and material convection limit the rate of thermal softening and lead to larger energy dissipation during the shear band evolution (Ye et al., 2012; Grady, 1992). Thus more energy could be dissipated by momentum diffusion when the workpiece material is more rate-sensitive and the chip flow is faster. This makes the stress release propagating to a farther distance during the process of serrated chip formation, and hence renders larger segment spacing.

\section{Conclusion remarks}

High speed machining was undertaken using a Ti-6Al-4V alloy with cutting speeds ranging from 0.05 to $31.2 \mathrm{~m} / \mathrm{s}$. The microscopic observations of chips reveal that the transition of chip morphology from continuous to serrated can be attributed to a repeated adiabatic shear band formation in PSZ, and the shear band could evolve to a higher level as the cutting speed increases. Based on the experimental observations, a theoretical model, taking into account the shear band evolution and material convection, was developed to predict the segment spacing. In order to verify this theoretical model, the FEM simulation, which was validated by cutting experiments, was further carried out to estimate the evolution degree of shear band. The predicted segment spacing is in good agreements with the experimental/simulated results, which shows the validity of this momentum diffusion-based model. Moreover, this theoretical model was compared with the WO, Molinari and GK models which were proposed for the spacing prediction of the multiple adiabatic shear bands. It seems that the model based on the perturbation analysis gives the lower bound for the segment spacing, while the model proposed from the momentum diffusion for maturely developed shear band gives the upper one. Finally, the roles of the material rate-hardening and material convection were also discussed, clearly showing that both of them render larger segment spacing.

\section{Acknowledgments}

This work has been supported by the Nature Science Foundation of China (Grants Nos. 11132011, 11021262 and 11002144), the National Basic Research Program of China (Grant Nos: 2012CB937500 and 2009CB724401), and the National Natural Science Foundation of China-NSAF. Grant No: 10976100.

\section{References}

Aifantis, E.C., 1987. The physics of plastic deformation. International Journal of Plasticity 3, 211-247.

Amin, A.K.M.N., Ismail, A.F., Khairusshima, M.K.N., 2007. Effectiveness of uncoated WC-Co and PCD inserts in end milling of titanium alloy - Ti-6Al-4V. Journal of Materials Processing Technology 192-193, 147-158.

Anand, L., Aslan, O., Chester, S.A., 2012. A large-deformation gradient theory for elastic - plastic materials: strain softening and regularization of shear bands. International Journal of Plasticity 30-31, 116-143.

Anderson, T.L., 2005. Fracture Mechanics, third ed. Taylor \& Francis, Boca Raton.

Arrazola, P.J., Villar, A., Ugarte, D., Marya, S., 2007. Serrated chip prediction in finite element modeling of the chip formation process. Machining Science and Technology 11, 367-390. 
Arrazola, P.J., Barbero, O., Urresti, I., 2010. Influence of material parameters on serrated chip prediction in finite element modeling of chip formation process. The International Journal of Material Forming 3, 519-522.

Astakhov, V.P., 1998. Metal Cutting Mechanics. USA CRC Press, Boca Raton.

Astakhov, V.P., 2010. Geometry of Single-Point Turning Tools and Drills. Springer London Ltd, London.

Atkins, A.G., 2003. Modelling metal cutting using modern ductile fracture mechanics quantitative explanations for some long standing problems. International Journal of Mechanical Sciences 45, 373-396.

Atkins, A.G., 2009. The Science and Engineering of Cutting: The Mechanics and Processes of Separating, Scratching and Puncturing Biomaterials, Metals and Non-Metals. Butterworth-Heinemann, London.

Bai, Y.L., Dodd, B., 1992. Adiabatic Shear Localization. Pergamon Press, Oxford.

Bäker, M., 2006. Finite element simulation of high-speed cutting forces. Journal of Materials Processing Technology 176, $117-126$.

Barry, J., Byrne, G., 2002. The mechanisms of chip formation in machining hardened steel. Journal of Manufacturing Science and Engineering, Transactions of ASME 124, 528-535.

Batra, R.C., Wei, Z.G., 2006. Shear band spacing in thermoviscoplastic materials. International Journal of Impact Engineering 32, 947-967.

Burns, T.J., Davies, M.A., 1997. Nolinear dynamics model for chip segmentation in machining. Physical Review Letters 79, 447-450.

Burns, T.J., Davies, M.A., 2002. On repeated adiabatic shear band formation during high-speed machining. International Journal of Plasticity 18, 487-506.

Calamaz, M., Coupard, D., Girot, F., 2008. A new material model for 2D numerical simulation of serrated chip formation when machining titanium alloy Ti6Al-4V. International Journal of Machine Tools and Manufacture 48, 275-288.

Chen, G., Ren, C.Z., Yang, X.Y., Jin, X.M., Guo, T., 2011. Finite element simulation of high-speed machining of titanium alloy (Ti-6Al-4V) based on ductile failure model. International Journal of advanced Manufacture Technology 56, 1027-1038.

Chen, K.W., Lin, J.F., 2010. Investigation of the relationship between primary and secondary shear bands induced by indentation in bulk metallic glasses. International Journal of Plasticity 26, 1645-1658.

Childs, T., Maekawa, K., Obikawa, T., Yamane, Y., 2000. Metal Machining: Theory and Applications. Arnold Publishers, London.

Cotterell, M., Byrne, G., 2008. Dynamics of chip formation during orthogonal cutting of titanium alloy Ti-6Al-4V. CIRP Annals 57, 93-96.

Davies, M.A., Burns, T.J., Evans, C.J., 1997. On the dynamics of chip formation in the machining of hard metals. CIRP Annals 46, 25-30.

Davies, M.A., Chou, Y., Evans, C.J., 1996. On chip morphology, tool wear and cutting mechanics in finish hard turning. CIRP Annals 45, 77-82.

Elbestawi, M.A., Srivastava, A.K., El-Wardany, T.I., 1996. A model for chip formation during machining of hardened steel. CIRP Annals 45, 71-76.

Farrokh, B., Khan, A.S., 2009. Grain size, strain rate, and temperature dependence of flow stress in ultra-fine grained and nanocrystalline Cu and Al: Synthesis, experiment, and constitutive modeling. International Journal of Plasticity 25, 715-732.

Grady, D.E., 1992. Properties of an adiabatic shear-band process zone. Journal of the Mechanics and Physics of Solids 40, 1197-1215.

Grady, D.E., Kipp, M.E., 1987. The growth of unstable thermoplastic shear with application to steady-wave shock compression in solids. Journal of the Mechanics and Physics of Solids 35, 95-118.

Henann, D.L., Anand, L., 2009. Fracture of metallic glasses at notches: effects of notch-root radius and the ratio of the elastic shear modulus to the bulk modulus on toughness. Acta Materialia 57, 6057-6074.

Hortig, C., Svendsen, B., 2007. Simulation of chip formation during high-speed cutting. Journal of Materials Processing Technology 186 , 66-76.

Huang, J., Aifantis, E.C., 1997. A note on the problem of shear localization during chip formation in orthogonal machining. Journal of Materials Engineering and Performance 6, 25-26.

Huang, J., Kalaitzidou, K., Sutherland, J.W., Aifantis, E.C., 2007. Validation of a predictive model for adiabatic shear band formation in chips produced via orthogonal machining. Journal of Mechanical Behavior Materials 18, 243-263.

Ikeda, M., Komatsu, S.Y., Sugimoto, T., Hasegawa, M., 1998. Effect of two phase warm rolling on aging behavior and mechanical properties of Ti-15Mo-5Zr3Al alloy. Materials Science and Engineering A 243, 140-145.

Jiang, M.Q., Dai, L.H., 2009a. On the origin of shear banding instability in metallic glasses. Journal of the Mechanics and Physics of Solids 57, 1267-1292.

Jiang, M.Q., Dai, L.H., 2009b. Formation mechanism of lamellar chips during machining of bulk metallic glass. Acta Materialia $57,2730-2738$.

Jiang, M.Q., Dai, L.H., 2011. Shear band toughness of bulk metallic glasses. Acta Materialia 59, $4525-4537$.

Khan, A.S., Liu, H., 2012a. A new approach for ductile fracture prediction on Al 2024-T351 alloy. International Journal of Plasticity $35,1-12$.

Khan, A.S., Liu, H., 2012b. Strain rate and temperature dependent fracture criteria for isotropic and anisotropic metals. International Journal of Plasticity 37, $1-15$.

Kobayashi, M., 2010. Analysis of deformation localization based on proposed theory of ultrasonic wave velocity propagating in plastically deformed solids. International Journal of Plasticity 26, 107-125.

Komanduri, R., 1982. Some clarifications on the mechanisms of chip formation when machining titanium alloys. Wear 76, $15-34$.

Komanduri, R., 1984. Shear-localization machining. Journal of Metals 36, 59.

Kumar, M.A., Mahesh, S., 2012. Banding in single crystals during plastic deformation. International Journal of Plasticity 36, 15-33.

Kuroda, M., 1996. Roles of plastic spin in shear banding. International Journal of Plasticity 12, 671-693.

Kuroda, M., Tvergaard, V., 2007. Effects of texture on shear band formation in plane strain tension/compression and bending. International Journal of Plasticity 23, 244-273.

Lee, W.S., Lin, C.F., 1998. High-temperature deformation behaviour of Ti6Al4V alloy evaluated by high strain-rate compression tests. Journal of Materials Processing Technology 75, 127-136.

Liang, R., Khan, A.S., 1999. A critical review of experimental results and constitutive models for BCC and FCC metals over a wide range of strain rates and temperatures. International Journal of Plasticity 15, 963-980.

Luo, M., Dunand, M., Mohr, D., 2012. Experiments and modeling of anisotropic aluminum extrusions under multi-axial loading - part II: ductile fracture. International Journal of Plasticity 32-33, 36-58.

Ma, W., Li, X.W., Dai, L.H., Ling, Z., 2012. Instability criterion of materials in combined stress states and its application to orthogonal cutting process. International Journal of Plasticity 30-31, 18-40.

Merchant, M.E., 1945. Mechanics of the metal cutting process I: orthogonal cutting and the type 2 chip. Journal of Applied Physics 16 , $267-275$.

Meredith, C.S., Khan, A.S., 2012. Texture evolution and anisotropy in the thermo-mechanical response of UFG Ti processed via equal channel angular pressing. International Journal of Plasticity 30-31, 202-217.

Meyers, M.A., 1994. Dynamic Behavior of Materials. Wiley, New York.

Meyers, M.A., Nesterenko, V.F., LaSalvia, J.C., Xue, Q., 2001. Shear localization in dynamic deformation of materials: microstructural evolution and selforganization. Materials Science and Engineering A 317, 204-225.

Molinari, A., 1997. Collective behavior and spacing of adiabatic shear bands. Journal of the Mechanics and Physics of Solids 45, 1551-1575.

Molinari, A., Dudzinski, D., 1992. Stationary shear band in high-speed machining. Comptes Rendus del Academie des Sciences Serie II 315, 399-405.

Molinari, A., Musquar, C., Sutter, G., 2002. Adiabatic shear banding in high speed machining of Ti-6AL-4V: experiments and modeling. International Journal of Plasticity 18, 443-459.

Mroginski, J.L., Etse, G., Vrech, S.M., 2011. A thermodynamical gradient theory for deformation and strain localization of porous media. International Journal of Plasticity 27, 620-634.

Nakayama, K., Arai, M., Kada, T., 1988. Machining characteristics of hard materials. CIRP Annals 37, 89-92.

Oxley, P.L.B., 1989. Mechanics of Machining: An Analytical Approach to Assessing Machinability. Wiley, New York.

Recht, R.F., 1964. Catastrophic thermoplastic shear. Transaction of ASME 86, 186-193.

Rittel, D., Wang, Z.G., Merzer, M., 2006. Adiabatic shear failure and dynamic stored energy of cold work. Physical Review Letters $96,075502$.

Rittel, D., Landau, P., Venkert, A., 2008. Dynamic recrystallization as a potential cause for adiabatic shear failure. Physical Review Letters $101,165501$. 
Schulz, H., Moriwaki, T., 1992. High-speed machining. CIRP Annals 41, 637-642.

Semiatin, S.L., Rao, S.B., 1983. Shear localization during metal cutting. Materials Science and Engineering 61, $185-192$.

Shaw, M.C., 2005. Metal Cutting Principles, second ed. Oxford University Press, Oxford.

Shaw, M.C., Vyas, A., 1993. Chip formation in the machining of hardened steel. CIRP Annals 42, 29-33.

Sheikh-Ahmad, J.Y., Quarless, V., Bailey, J.A., 2004. On the role of microcracks on flow instability in low speed machining of CP titanium. Machining Science and Technology 8, 415-430.

Sun, S., Brandt, M., Dargusch, M.S., 2009. Characteristics of cutting forces and chip formation in machining of titanium alloys. International Journal of Machine Tools and Manufacture 49, 561-568.

Sutter, G., Molinari, A., 2005. Analysis of the cutting force components and friction in high speed machining. Journal of Manufacturing Science and Engineering 127, 245-250.

von-Turkovich, B.F., Durham, D.R., 1982. Machining titanium and its alloys. In: Advanced Processing Methods for Titanium, Metallurgical Society of AIME Conference, pp. 257-274.

Vyas, A., Shaw, M.C., 1999. Mechanics of saw-tooth chip formation in metal cutting. Journal of Manufacturing Science and Engineering $121,163-172$.

Wang, H., To, S., Chan, C.Y., Cheung, C.F., Lee, W.B., 2010. A study of regularly spaced shear bands and morphology of serrated chip formation in microcutting process. Scripta Materialia 63, 227-230.

Wang, H., To, S., Chan, C.Y., Cheung, C.F., Lee, W.B., 2011. Dynamic modelling of shear band formation and tool-tip vibration in ultra-precision diamond turning. International Journal of Machine Tools and Manufacture 51, 512-519.

Wright, T.W., Ockendon, H., 1996. Research note: a scaling law for the effect of inertial on the formation of adiabatic shear bands. International Journal of Plasticity 12, 927-934.

Wu, F.F., Zheng, W., Wu, S.D., Zhang, Z.F., Shen, J., 2011. Shear stability of metallic glasses. International Journal of Plasticity $27,560-575$.

Xue, Q., Meyers, M.A., Nesterenko, V.F., 2002. Self-organization of shear bands in titanium and Ti-6Al-4V alloy. Acta Materialia 50, $575-596$.

Xue, Q., Meyers, M.A., Nesterenko, V.F., 2004. Self organization of shear bands in stainless steel. Materials Science and Engineering A $384,35-46$.

Ye, G.G., Xue, S.F., Ma, W., Jiang, M.Q., Ling, Z., Tong, X.H., Dai, L.H., 2012. Cutting AISI 1045 steel at very high speeds. International Journal of Machine Tools and Manufacture 56, 1-9.

Zbib, H.M., Aifantis, E.C., 1988. On the localization and postlocalization behavior of plastic deformation - I/II/III. Review of Mechanics 23, $261-305$.

Zbib, H.M., Aifantis, E.C., 1992. On the gradient-dependent theory of plasticity and shear banding. Acta Mechanica 92, $209-225$.

Zbib, H.M., Aifantis, E.C., 2003. Size effects and length scales in gradient plasticity and dislocation dynamics. Scripta Materialia 48, 155-160.

Zbib, H.M., Jubran, J.S., 1992. Dynamic shear banding: a three-dimensional analysis. International Journal of Plasticity 8, 619-641.

Zhou, F., Wright, T.W., Ramesh, K.T., 2006. The formation of multiple adiabatic shear bands. Journal of the Mechanics and Physics of Solids 54, $1376-1400$. 